\title{
El impacto de la tableta en la educación primaria
}

\author{
The impact of the tablet in primary education
}

\author{
Cristina Sánchez-Martínez, María-Carmen Ricoy \\ Universidad de Vigo
}

\begin{abstract}
Resumen
Las dotaciones tecnológicas con las que cuentan las instituciones reflejan la voluntad de promover, desde la escuela, la incorporación de los avances digitales. Ante la demanda emergente, la finalidad general de esta investigación es analizar la utilización de la tableta en la Educación Primaria. En este trabajo se analizan las noticias de prensa que muestran la difusión de experiencias con este dispositivo. La metodología de investigación utilizada es de tipo cualitativo. Como conclusiones preliminares, cabe indicar que el impacto de la tableta es sutil, aunque son variados los beneficios que se le atribuye, a pesar de desprender también algún inconveniente.
\end{abstract}

Palabras clave: Tableta, Educación Primaria, aprendizaje.

\begin{abstract}
Technological capabilities which will include institutions reflect the desire to promote from school, the incorporation of digital progress. In the emerging demand, the general purpose of this research is to analyze the use of the tablet in Primary Education. In this paper the news release showing the sharing of experiences with this device are analyzed. The research methodology is qualitative. As preliminary conclusions, it should be noted that the impact of the tablet is subtle, though many benefits attached to it, although also release any problems.

Keywords: Tablet, primary education, learning.
\end{abstract}

En la actualidad diversos centros de nuestra Comunidad, así como otros del resto del Estado y de otros países, llevan a cabo la implantación de distintas experiencias curriculares con las tabletas. De hecho la tableta es uno de los dispositivos en boga en las escuelas y universidades (Feliz-Murias, Ricoy y Feliz, 2014). Estos recursos, de forma cotidiana, ya se venían utilizando en múltiples situaciones y espacios. De hecho las tabletas han irrumpido con fuerza en España, país que duplica a Europa en número de usuarios; ya que frente al $14 \%$ de media en nuestro país, en Europa se registra el 7\% (Informe Mobile Life, 2012).

Entre otros aparatos digitales, las tabletas facilitan el desarrollo de multitud de prácticas e incentivan el proceso de aprendizaje. Las herramientas tecnológicas, y entre ellas las tabletas, posibilitan la realización de actividades cooperativas, la potenciación del desarrollo de habilidades cognitivas y la adquisición de nuevas competencias. De hecho siguiendo a Guo et. al. (2015) las tabletas representan un sustituto adecuado a la metodología didáctica tradicional apoyada básicamente en el papel y lápiz.

El alumnado que utiliza la tableta manifiesta cierta preferencia y entusiasmo respondiendo a las demandas escolares. Haciéndose eco de ello, algunos centros escolares ya llevan a cabo proyectos focalizando el proceso de enseñanza con las tabletas. Muestra de esto es el Colegio Bilingüe Liceo Sorolla de Pozuelo de Alarcón en Madrid (Liceo Sorolla, 2015), que ponen de manifiesto que el $86 \%$ del alumnado está más contento en clase desde que trabaja con la tableta; mientras el $67 \%$ afirma ser más creativo. Además, es uno de los recursos emergentes que estimula la innovación pedagógica.

Muchas de las investigaciones actuales, sobre la integración curricular de las tabletas, apuntan que su uso escolar mejora el aprendizaje del alumnado al incrementar su nivel de atención (Koile y Singer, 2006); $\mathrm{y}$ agiliza el feedback entre estudiante y profesorado (Enríquez, 2010). En esta línea, cabe indicar que en general la tecnología ha tenido un impacto significativo en la forma en que los-as docentes enseñan y en la que el alumnado aprende (Massingham y Herrington, 2006).

\section{Métodos}

El estudio realizado forma parte de una investigación más amplia sobre el uso de las tabletas en la Educación Primaria. La presente aportación se encuadra en la investigación cualitativa y es de carácter exploratorio. La metodología cualitativa, como indica su propia denominación, tiene como objetivo la descripción de las cualidades de una situación o fenómeno, así como su análisis. No trata de probar o de medir el grado de una cualidad, sino que intenta descubrir tantas atribuciones como sea posible (Mendoza, 2006).

A partir del tópico objeto de estudio, la finalidad principal de esta investigación es conocer el impacto de la tableta en el alumnado de Educación Primaria descubriendo sus beneficios y controversias. En concreto, en este trabajo se indaga sobre distintas experiencias en las que se integra la tableta en la etapa de E. Primaria. Las preguntas centrales de investigación son las siguientes:

- ¿Dónde y cuándo se utilizan las tabletas?

- ¿Quién promueve la dotación de las tabletas en los centros educativos?

- ¿Cuáles son las ventajas e inconvenientes de utilizar la tableta en el proceso de aprendizaje?

\section{Muestra}

La muestra de estudio está configurada por 30 noticias de periódicos nacionales e internacionales, que difundieron experiencias sobre las tabletas sobre el alumnado de Educación Primaria. Esta información fue extraída de distintos diarios con acceso libre y gratuito. 
El año de publicación con mayor producción se sitúa en $2015(f=13)$, seguido del $2014(f=7)$. En 2009 y 2011 se han localizado menos publicaciones sobre la temática (tabla 1).

Tabla1.

Identificación de la muestra

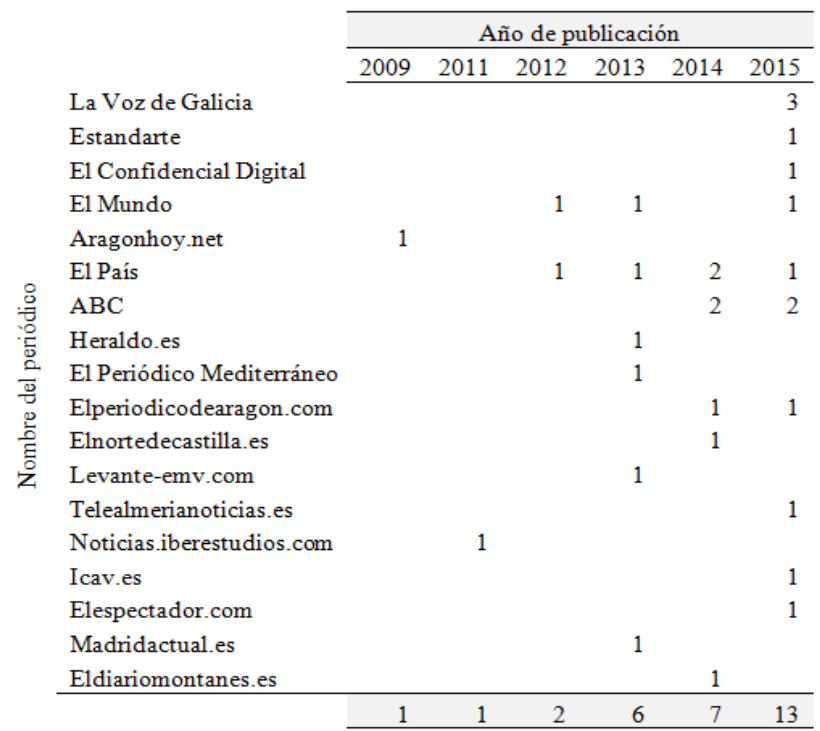

\section{Materiales}

La recogida de información se centró principalmente en la exploración de noticias de periódicos de tirada autonómica, nacional e internacional. Esta selección se focalizó en la identificación de noticias sobre el uso de la tableta en centros de Educación Primaria. La mayor parte de las noticias fueron extraídas de periódicos digitales de acceso libre. Su localización se realizó a través del buscador de google y en otros casos del buscador interno del propio periódico.

\section{Procedimiento de análisis}

Para analizar la información recogida se utilizó el programa de Analysis of Qualitative Data (AQUAD), versión 7. Los textos fueron examinados en su integridad mediante un análisis de contenido. En la delimitación de las categorías se han considerado diferentes aspectos sobre la implantación de las tabletas en las aulas de Educación Primaria.

Para garantizar la consistencia de los resultados y conclusiones obtenidos se contemplaron cuidadosamente todos los elementos del diseño y metodología de investigación. En cuanto al proceso de análisis, cabe resaltar la rigurosidad que permite el programa AQUAD (Ricoy y Fernández, 2011). De hecho, Bensing et al. (2011) subrayan que los datos deben ser codificados e interpretados de forma fiel.

\section{Resultados}

En este apartado se exponen los resultados obtenidos, a través de distintos subapartados, y se ilustran con varios fragmentos extraídos de las noticias de prensa escrita, así como con diferentes figuras. Estos resultados procedentes del análisis de contenido se han reunido alrededor de 4 ejes vinculados con las cuestiones de investigación.
Tipología de centro, localidad y período. Partiendo de las dos tipologías de centro dominantes (públicos y privados) se han extraído los resultados al respecto (figura 1).

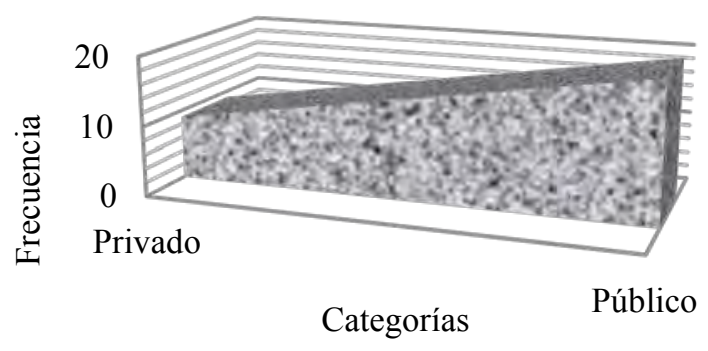

Figura 1. Tipología de centro

De los resultados obtenidos se desprende que predomina la incorporación de las tabletas en los centros públicos $(\mathrm{f}=20)$, frente a los centros privados $(\mathrm{f}=9)$. Las noticias de los propios periódicos (La Voz de Galicia, El Estandarte, El País, entre otros) reafirman este dato poniendo de manifiesto la superioridad de los centros de carácter público con el uso de las tabletas, respecto a los de tipo privado.

En cuanto al contexto escolar en el que tiene lugar la implementación de las tabletas, en los centros de E. Primaria analizados, sobresale la comunidad gallega y la Valenciana. En contraposición se desprende, de los análisis realizados, que apenas se encontraron evidencias del uso de la tableta en la comunidad de: Cantabria, Cataluña, País Vasco. Del mismo modo también resulta excepcional su revelación en el contexto de Colombia. Cabe resaltar que muchas de las noticias de prensa tenían carácter autonómico, y se encontraban en la sección comarcal.

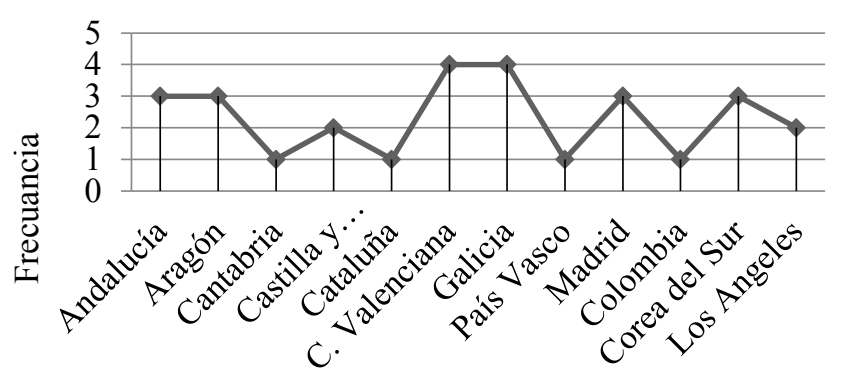

Categorías

Figura 2. Comunidades de implementación de experiencias con la tableta.

A modo de ejemplo se aporta el siguiente extracto:

Colombia hace su mayor inversión tecnológica en la escuela pública (El País, marzo 2015. Líneas 12-13).

Las noticias analizadas ponen de manifiesto que la mayor difusión de experiencias sobre la implementación de la tableta se supedita al curso escolar 2013-2014 ( $\mathrm{f}=9$ ), así como al 2014-2015 ( $\mathrm{f}=8)$. En contraposición, los cursos en los que se detecta un menor número de noticias sobre este dispositivo fueron el 2009-2010 y el 2012-2013. 


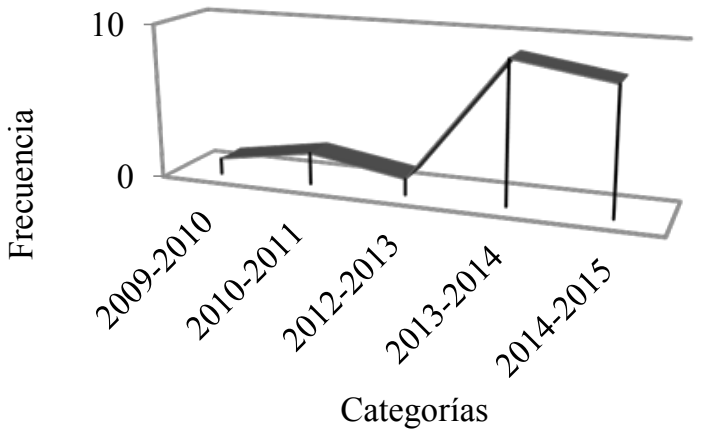

Figura 3. Curso académico de desarrollo de experiencias con la tableta

Los cursos de Educación Primaria en los que la prensa refleja que se están introducido o se han usado las tabletas se supeditan al de (figura 4): $5^{\circ}$ de E. Primaria $(\mathrm{f}=14), 6^{\circ}$, y en general a los demás cursos $(\mathrm{f}=20)$. Por su notoriedad cabe referirse a su implantación con el alumnado de $5^{\circ}$ curso. A partir de alguna noticia de las analizadas, se pone de manifiesto que los escolares de la educación obligatoria utilizan los dispositivos móviles y los materiales digitales en el contexto educativo. Este es un primer paso para la renovación o para la innovación pedagógica.

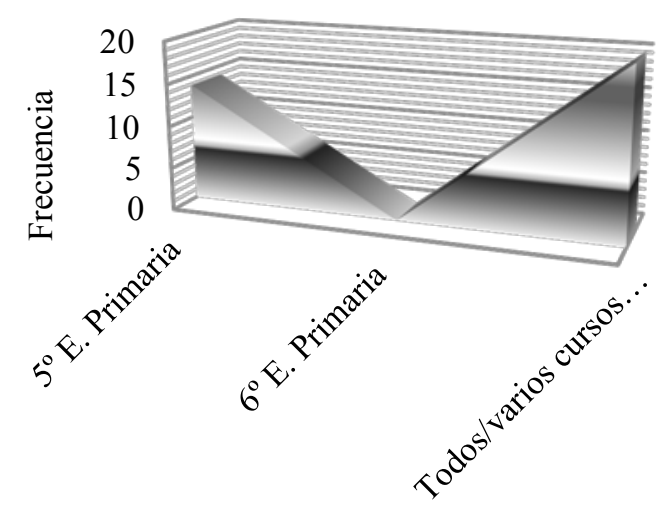

Categorías

Figura 4. Cursos con experiencias sobre la tableta

De forma ilustrativa se ofrecen los siguientes textos: Los alumnos de $5^{\circ}$ del colegio público Montecorona de Sabiñánigo ya estudian con tableta y materiales digitales (Heraldo.es, noviembre 2013. Línea 31-33) Cuatro comunidades autónomas, Cataluña, Andalucía, Aragón y Madrid, ya han iniciado el paso a la mochila digital con el uso de las tablets que prevé implantar voluntariamente en $5^{\circ}$ de Primaria este próximo curso la Consellería de Educación en Castellón y toda la Comunitat. (El periódico mediterráneo, junio 2013. Línea 32-38).

Dotación de las tabletas en los centros. Cabe mencionar la contribución en infraestructuras y dispositivos digitales de los ayuntamientos, empresas, entidades bancarias, gobiernos autonómicos, internacionales y gobiernos nacionales (figura 5).

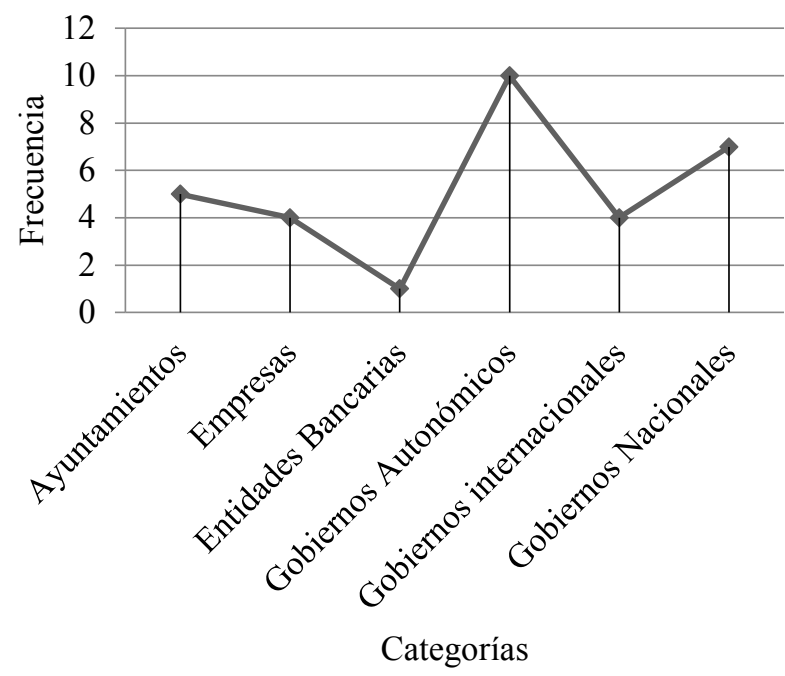

Figura 5. Dotación de tabletas por la administración u otras entidades

A partir de la información extraída de la prensa se detecta una valiosa dotación de tabletas a los centros educativos por los gobiernos autonómicos $(\mathrm{f}=10)$ y nacionales $(\mathrm{f}=7)$. Por otra parte, de forma excepcional se revela la subvención realizada por una entidad bancaria. Tal y como se afirma en el periódico ABC, la Junta de Andalucía repartiría un número elevado de tabletas, a un coste módico rentabilizando de este modo la asignación económica de los centros de Educación Primaria. De las noticias de periódico analizadas se desprende que la comunidad de Madrid dedica el triple de presupuesto a este tipo de iniciativas, y le pone como requisito a los centros escolares para su participación que se impliquen, al menos, la mitad de sus docentes. Véanse los siguientes extractos:

La Junta (de Andalucía) repartirá casi 30.000 tabletas de 283 euros en los colegios (ABC, enero 2015. Línea 16-17).

(...). Como hemos señalado, cada centro cuenta con una dotación de hasta 100.000 euros para el desarrollo de su proyecto que, si bien ha comenzado este curso, tiene una duración de tres cursos escolares. La dotación completa para estos centros elegidos es, aproximadamente, de un millón de euros.

Como requisito para participar en la convocatoria, cada proyecto debía contar con, al menos, la participación del 50 por ciento de los profesores del centro. Lo más agradable para la Comunidad de Madrid ha sido comprobar que el 86 por ciento de los proyectos presentados venía avalado con un apoyo superior al 70 por ciento del claustro de profesores. (ABC, abril 2015. Línea 108-123).

Beneficios y controversias del uso de la tableta. Teniendo en cuenta la información extraída de las noticias de prensa (figura 6), los principales beneficios del uso de la tableta están asociados al ahorro económico que supone para la administración y la familia; así como al enriquecimiento académico y la actualización posibilitada. Cabe destacar que, según los periódicos, las familias del alumnado están de acuerdo con que sus 
hijos/as participen en proyectos educativos que utilizan la tableta.

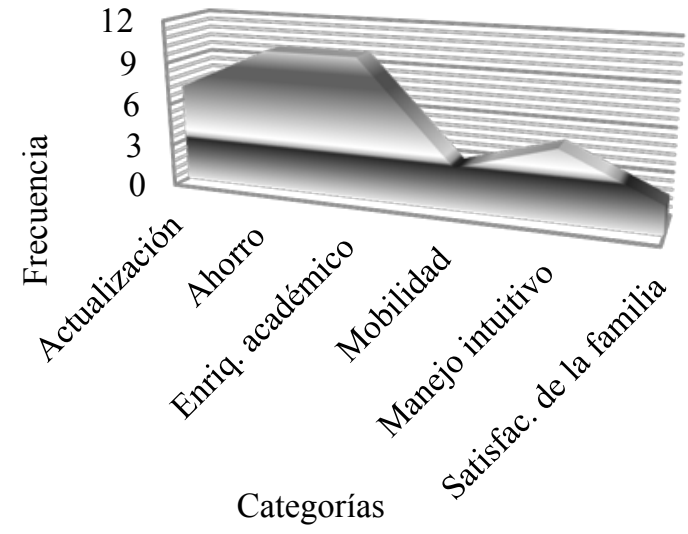

Figura 6. Beneficios asociados al uso de la tableta

De modo ilustrativo se ofrece el siguiente extracto:

Aunque la compra es voluntaria, Maseda asegura que todos los padres han estado de acuerdo en involucrar a sus hijos en un proyecto educativo que se extiende en Barbanza (La Voz de Galicia, enero 2015. Línea 56-60).

¿Son realmente beneficiosas para los alumnos?

Almudena Castellanos, doctora en Ciencias de la Educación y profesora de Tecnología Educativa de la Universidad Internacional de La Rioja (UNIR), destaca que la principal ventaja del uso de la tableta radica precisamente en lo intuitivo que es su manejo. Con un solo dedo abrimos carpetas, agrandamos.(Estandarte, septiembre 2013 Línea 44-51).

Tal como plasman las noticias de prensa, la tableta al igual que otros dispositivos táctiles es un recurso muy intuitivo y fácil de manejar. Esto es debido a sus características táctiles y que reacciona al tocarle, por lo que no se necesitan de entrada muchas competencias digitales ni movimientos motrices. Además no requiere el uso de periféricos para su manejo básico.

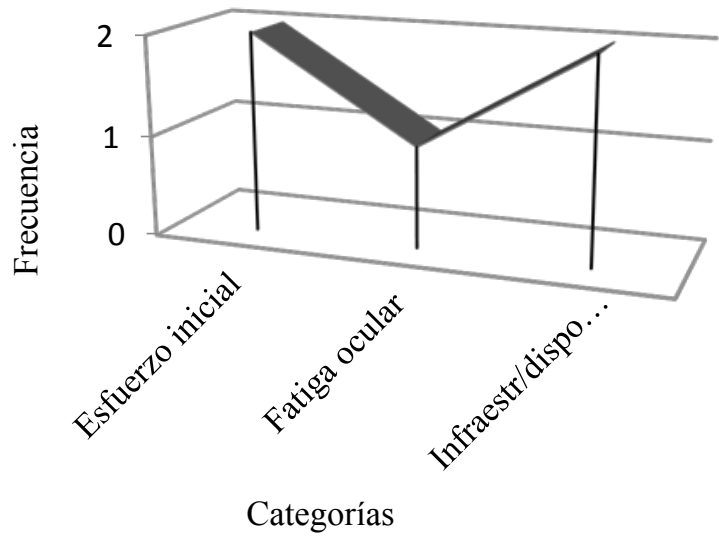

Figura 7. Controversias vinculadas a la tableta

Los principales problemas o controversias del uso de las tabletas, en las que inciden las noticias de prensa, se asocian con el esfuerzo inicial que exigen, la escasez de dispositivos existentes en los centros educativos. En este sentido se refieren por ejemplo, como afirma el periódico la Voz de Galicia, a la poca velocidad de Internet y la dependencia del suministro de la batería y su fragilidad en general; además cabe considerar las propias preferencias por la lectura en papel frente a los inconvenientes del formato digital. En este sentido, de forma excepcional se apunta la fatiga ocular resaltando sus consecuencias negativas.

Con todo, en algunos de los centros la velocidad de Internet no es la idónea. (La Voz de Galicia, enero 2015. Línea 70-71).

Tiene desventajas, claro. «Que te duelen los ojos después de mucho rato», dice una estudiante. Y que hay que tener cuidado con la batería y con el material, y que para estudiar es mejor «leer en papel», asegura otro chico. (Elnortedecastilla.es, octubre 2014. Línea 165-169).

\section{Discusión y conclusiones}

En la actualidad la incorporación de las tabletas a las aulas de Educación Primaria es una realidad. Los centros públicos llevan ventaja respecto de los privados con la anexión de las tabletas. Esto está directamente relacionado con la dotación de infraestructuras y dispositivos, ya que son en gran medida, los gobiernos autonómicos los que hacen el mayor aporte económico que sostiene estos proyectos.

En un informe sobre América Latina, se reconoce el buen entendimiento, en aras de la gobernanza, entre los estados y el sector privado de las TIC (Lugo et. Al, 2014); al tiempo que las posibles discrepancias entre ambos se neutralizan mediante la proliferación de acreditaciones y evaluaciones que cada vez sean más intrusivas y punitivas, como da cumplida cuenta la LOMCE (2013). Quizás sea este el motivo de la existencia de las incipientes dotaciones.

A partir de las noticias analizadas, las localidades en las que más éxito está teniendo la implantación de las tabletas, en Educación Primaria, son las gallegas y las valencianas. Por otra parte, cabe indicar las iniciativas de países extranjeros como: Colombia, Corea del Sur y Los Ángeles. En general, y afortunadamente, se ubica la implantación de las tabletas en entornos dispares y desiguales. Lo que representa una muestra más del propio fenómeno mundial de globalización, aunque lamentablemente en algunos casos pueda venir a re-marcar las brechas de la desigualdad entre las personas.

El curso académico en el que se detecta una mayor implantación de las tabletas es el 2013-2014. Con todo, cabe indicar que se observa un crecimiento en su aplicación desde el curso 2009-2010 hasta el anterior.

La incorporación de la tableta se lleva a cabo en todos los cursos de E. Primaria, destacando $5^{\circ}$ curso. Probablemente este predominio se derive de la existencia de programas específicos de la administración que promueven el uso de tabletas o recursos TIC, siendo impulsados o respaldados principalmente por los gobiernos autonómicos.

Como principales beneficios asociados a la implementación de la tableta en la etapa de primaria, 
cabe destacar el ahorro y el enriquecimiento académico que suponen para los progenitores y para la administración. En contraposición, como controversia es de mencionar el esfuerzo inicial que genera el cambio, tanto por parte del alumnado, como del profesorado, así como las limitaciones que se desprenden, en ocasiones, de las propias carencias tecnológicas existentes en los centros escolares. De este modo se hace patente la brecha digital existente.

Por último resaltar, que tal como sostienen Feliz-Murias y Ricoy (2015), las tabletas continuaran evolucionando hacia modelos que hoy desconocemos y mejorarán mucho más sus características. De modo que sus posibilidades pedagógicas, previsiblemente, serán plenamente apreciadas en poco tiempo.

\section{Referencias}

Bensing, J., Deledda, G., Fletcher, I. Mazzi, M., Moretti, F., Rimondini, M. \& Van Vliet, L. (2011). A standardized approach to qualitative content analysis of focus group discussions from different countries. Classroom Interactions and Student Learning. Patient Education \& Counseling, 82, 420428.http://projects.csail.mit.edu/clp/publications/docu ments/KoileSinger06.pdf

Enríquez, A. (2010). Enhancing Student Performance Using Tablet Computers. College Teaching, 58(3), 77-84.

http://www.tandfonline.com/doi/abs/10.1080/8756755 0903263859

Feliz-Muria, T. \& Ricoy, M. C. (2015). La tableta: Fascinación por el aprendizaje ubicuo. En E. Vázquez-Cano \& M. L. Sevillano (Eds.), Dispositivos digitales móviles en educación: El aprendizaje ubicuo (pp. 85-103). Madrid: Narcea.

Feliz-Murias, T., Ricoy, C. \& Feliz, S. (2014). Innovative didactical strategies based on tablets and u-learning. En Actas de la conference proceedings the open and flexible higher education conference (pp. 142-156). Heerlen: EADTU. http://conference.eadtu.eu/images/Proceedings/Confer ence_2014___proceedings.pdf

Guo, Yi., Newell, S., Logan, H., Marks, J., Shepperd, J. (2015). Evaluating Tablet Computers as a Survey Tool in Rural Communities. Jorunal of Rural Health, 31,108-117.

Koile, K. \& Singer, D. (2006). The impact of pen-based technology on education; vignettes, evaluations $\mathrm{y}$ future directions. En Berque, D., Gray, J. \& Reed, R. (Ed.), Development of a tablet-PC-based system to increase instructor-student (pp. 70-87). EEUU: Purdue University Press.

Ley Orgánica 8/2013, de 9 de diciembre, para la mejora de la calidad educativa (LOMCE), BOE, 10 de diciembre de 2013.

Liceo Sorolla (2015). Learning with iPads at Liceo Sorolla.http://colegioliceosorolla.es/wp-content/uploa ds/2015/03/ESTUDIO-IPAD-LICEO-SOROLLA.pdf

Lugo, M. T., López, N. \& Toranzos, L. (2014). Políticas tic en los sistemas educativos de América Latina. Madrid: UNESCO-OEI.
Massingham, P. \& Herrington, T. (2006). Does attendance matter? An examination of student attitudes, participation, performance and attendance. Journal of University Teaching \& Learning Practice, $3(2)$, 83-103. http://ro.uow.edu.au/cgi/viewcontent.cgi?article $=1046$ \&context=jutlp

Mendoza, R. (2006). Investigación cualitativa y cuantitativa - Diferencias y Limitaciones. $\mathrm{http}: / /$ gycperu.com/descargas/005investigacion $\% 20 \mathrm{cu}$ ali\%20cuanti\%20diferencias\%20y\%20limitac.pdf

Ricoy, M. C. \& Fernández, J. (2011). Contribuciones y controversias que genera el uso de las TIC en la Educación Superior: un estudio de caso. Revista de Educación, 360, 509-532.

Ruíz, C. (2011). La investigación cualitativa en eduación: crítica y prospectiva. http://dialnet.unirioja.es/servlet/articulo? codigo $=4172$ 011

Sevillano \& Vázquez (2014). Análisis de la funcionalidad didáctica de las tabletas digitales en el espacio europeo de educación superior. Revista de Universidad y Sociedad del Conocimiento, 3(11). http://www.raco.cat/index.php/RUSC/article/viewFile $/ 285061 / 373077$

\section{Agradecimientos}

Aprovechamos para manifestar el agradecimiento a la Universidad de Vigo, por la financiación de esta investigación con el desarrollo de la Tesis Doctoral sobre el uso de las tabletas en la Educación Primaria. 